\title{
Review Article \\ The Advances of Carbon Nanotubes in Cancer Diagnostics and Therapeutics
}

\author{
Zhou Chen, ${ }^{1,2}$ Aili Zhang, ${ }^{3}$ Xiaobing Wang, ${ }^{4}$ Jing Zhu, ${ }^{5}$ Yamin Fan, \\ Hongmei Yu, ${ }^{7}$ and Zhaogang Yang ${ }^{6}$ \\ ${ }^{1}$ School of Mechanical and Power Engineering, Nanjing Tech University, Nanjing, Jiangsu 211800, China \\ ${ }^{2}$ Suzhou Yiheyongli New Energy Co., Ltd, Suzhou, Jiangsu 215400, China \\ ${ }^{3}$ Department of Cancer Biology, Cleveland Clinic, Cleveland, OH 44195, USA \\ ${ }^{4}$ Tumor Marker Research Center, Cancer Institute and Hospital, Chinese Academy of Medical Science and \\ Peking Union Medical College, Beijing 100021, China \\ ${ }^{5}$ College of Pharmacy, The Ohio State University, Columbus, OH 43210, USA \\ ${ }^{6}$ Department of Chemical and Biomolecular Engineering, The Ohio State University, Columbus, OH 43210, USA \\ ${ }^{7}$ China-Japan Union Hospital, Jilin University, Changchun, Jilin 130021, China
}

Correspondence should be addressed to Hongmei Yu; yuhongm@jlu.edu.cn and Zhaogang Yang; yzhaogang@gmail.com

Received 1 February 2017; Revised 8 May 2017; Accepted 14 May 2017; Published 9 August 2017

Academic Editor: Jian Zhong

Copyright (C) 2017 Zhou Chen et al. This is an open access article distributed under the Creative Commons Attribution License, which permits unrestricted use, distribution, and reproduction in any medium, provided the original work is properly cited.

\begin{abstract}
Carbon nanotubes (CNTs), one of the unique one-dimensional nanomaterials, have gained great attention because of their specific characters, versatile functionalization chemistry, and biological compatibility in the past few decades. CNTs can be functionalized via different methods to perform their specific functions. CNTs have been used in various areas of biomedicine as nanocarriers, including cancer diagnosis and therapy. Different molecules such as peptide, antigen, and nucleic acid can be delivered to cancer cells by CNTs with high efficiency. In this review, we summarized the properties of CNTs and the method of CNTs functionalization and illustrated their application in cancer diagnosis and therapy.
\end{abstract}

\section{Introduction}

Nanotechnology as one branch of engineering, which deals with the manipulation of individual molecules and atoms, has got much attention in recent years [1-8]. With the development of nanotechnology, many nanomaterials were developed and applied into industry [9-15]. Carbon nanotubes (CNTs) are one of the nanomaterials that are widely used in such area in recent years. They have got more and more attention because of their unique properties [16].

CNTs are more dynamic compared with other nanomaterials in their biological applications, and they are one of the most interesting nanocarriers in scientific studies. CNTs have displayed obvious prospect that they can cross the biological barriers as novel delivery systems. Compared with quantum dots which have been mainly used in cancer cell imaging, CNTs can also be applied in drug delivery and thermal ablation [17]. They have the ability to enter cells, and this behavior is independent of cell type and functional group at their surface. Currently the detailed mechanisms of internalization (endocytosis or needle like penetration) have not been completely explained [18-20]. The high area of CNTs can provide multiple sites for attachment of different molecules, which makes polyvalent derivatization possible. In addition, based on the in vitro and in vivo results obtained from different research groups, a variety of chemically functionalized CNTs have the ability of biocompatibility with the biological environment. These results emphasized how the behavior of this material could be regulated via the degree and type of functionalization in vivo, and these two aspects need to be precisely managed [21-27]. CNTs possess some characters, including a high interfacial area with cellular membrane and unique capacity to incorporate multiple functionalizations. At the same time, CNTs also have the compatibility and 


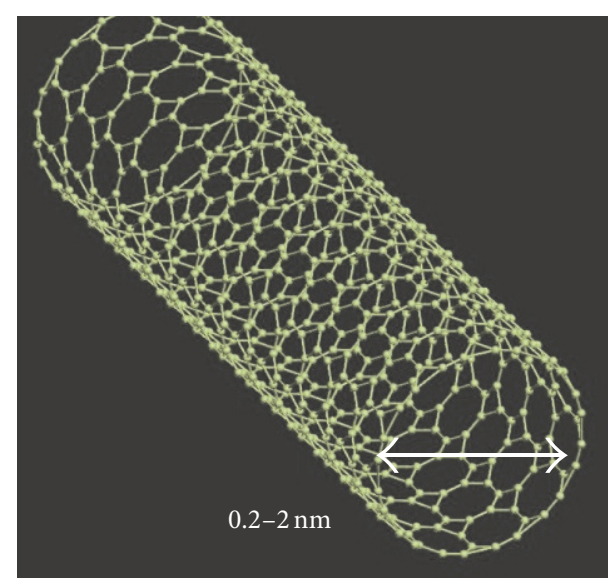

(a)

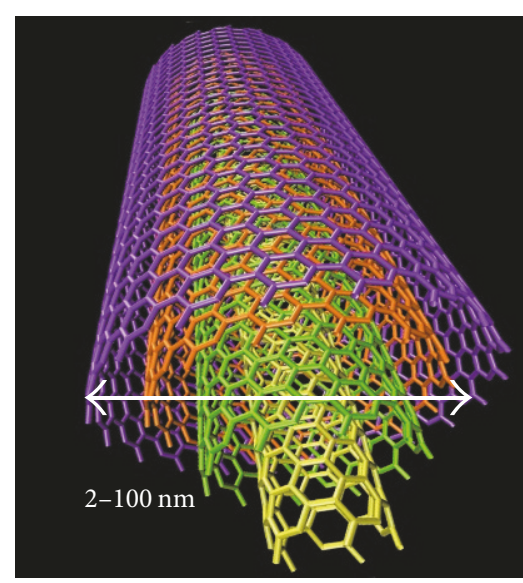

(b)

FIGURE 1: Conceptual diagrams of single-walled carbon nanotubes (SWCNTs) (a) and multiwalled carbon nanotubes (MWCNTs) (b).

transportability in biological fluids. These features make them a useful tool for all kinds of diagnosis and therapeutic as well as drug delivery application [28].

\section{Character and Performance Properties of CNTs}

CNTs were discovered in 1976 by Oberlin [29]. Currently, electric arc discharge, laser ablation, and thermal and plasma enhanced chemical vapor deposition (CVD) are the major technologies for the generating of CNTs [30]. CNTs can be divided into two broad classes based on their length, diameter, and structure. One is single-walled CNTs (SWCNTs) that consist of single sheet of cylindrical graphene, and the other is multiwalled carbon nanotubes (MWCNTs) which include several concentric graphene sheets [31, 32]. The structures of these two CNTs were indicated in Figure 1. Moreover, MWCNTs can be further divided into two subtypes: doublewalled CNTs (DWCNTs) and triple-walled CNTs (TWCNTs) [33]. MWCNTs consist of several concentric layers of rolled graphite; these concentric layers have an inner diameter of 1 to $3 \mathrm{~nm}$ and an outer diameter of 2 to $100 \mathrm{~nm}$ [34]. The thickness of single cylindrical carbon layer in SWCNTs is from 0.2 to $2 \mathrm{~nm}$ [35]. SWCNTs have a higher ratio of length than that of diameter. MWCNTs have wider inner diameter compared to SWCNTs, and this characteristic can offer more space for high drug loading. It has also been suggested that the outer shell of MWCNTs can be functionalized while at the same time the side wall was not damaged [36].

CNTs can be modified to improve their solubility by efficient methods, which can be employed in several biological applications [37]. CNTs can be oxidized by strong acids, which results in the decrease of length and production of carboxylic groups. And these changes can improve their solubility in watery solutions [38]. Alternatively, the external walls and tips can have better solubility via the extra reactions to the CNTs $[39,40]$. The solubility is a main property, which ensures the biocompatibility under physiological conditions [37]. Furthermore, virtually all kinds of active molecules can be linked to the functionalized carbon nanotubes ( $\mathrm{f}$ CNTs), including peptides, proteins, nucleic acids, and other therapeutic agents. The physicochemical properties of CNTs mainly associate with several aspects, including an organized architecture with a high aspect ratio, surface area, mechanical strength, electrical and thermal conductivity, metallic or semimetallic behavior, and ultra-lightweight [41]. These advantages make CNTs the hopeful candidate material that possess enormous biomedical potentials [42]. However pristine CNTs (such as prepared and nonfunctionalized) are inherently hydrophobic, which have limited biomedical applications. Moreover, the nonfunctionalized CNTs exhibit high cytotoxicity, and it may be due to the insolubility of CNTs or the residual metal catalysts in CNTs. The toxicity of CNTs requires their functionalization, rendering them soluble and biocompatible so that they can be integrated into a biological system. Many molecules can be bound to CNTs (Figure 2) [31].

\section{The Techniques of CNTs Functionalization}

Even though CNTs have many advantages, their biomedical developments were limited. The purification of CNTs is still not well developed. Meanwhile, CNTs are difficult to dissolve in aqueous media, which limits their biomedical application, although this disadvantage can be overcome through functionalization [43]. In addition, CNTs are usually undissolved, and they also do not have good biocompatibility. In order to increase their biosolubility, two main techniques such as noncovalent functionalization and covalent functionalization are currently widely used. The covalently or noncovalently functionalized CNTs can be achieved via using different chemical groups [44]. CNTs can be divided into two zones according to their reactivity with functional groups, including tips and side walls. The tips are considered to have stronger functional groups bonding capabilities than the side walls.

3.1. Noncovalent Functionalization. Using the advantage of the conservation of the electronic structure of the nanotube aromatic, surfactants, peptide, polymer, nucleic acids, 


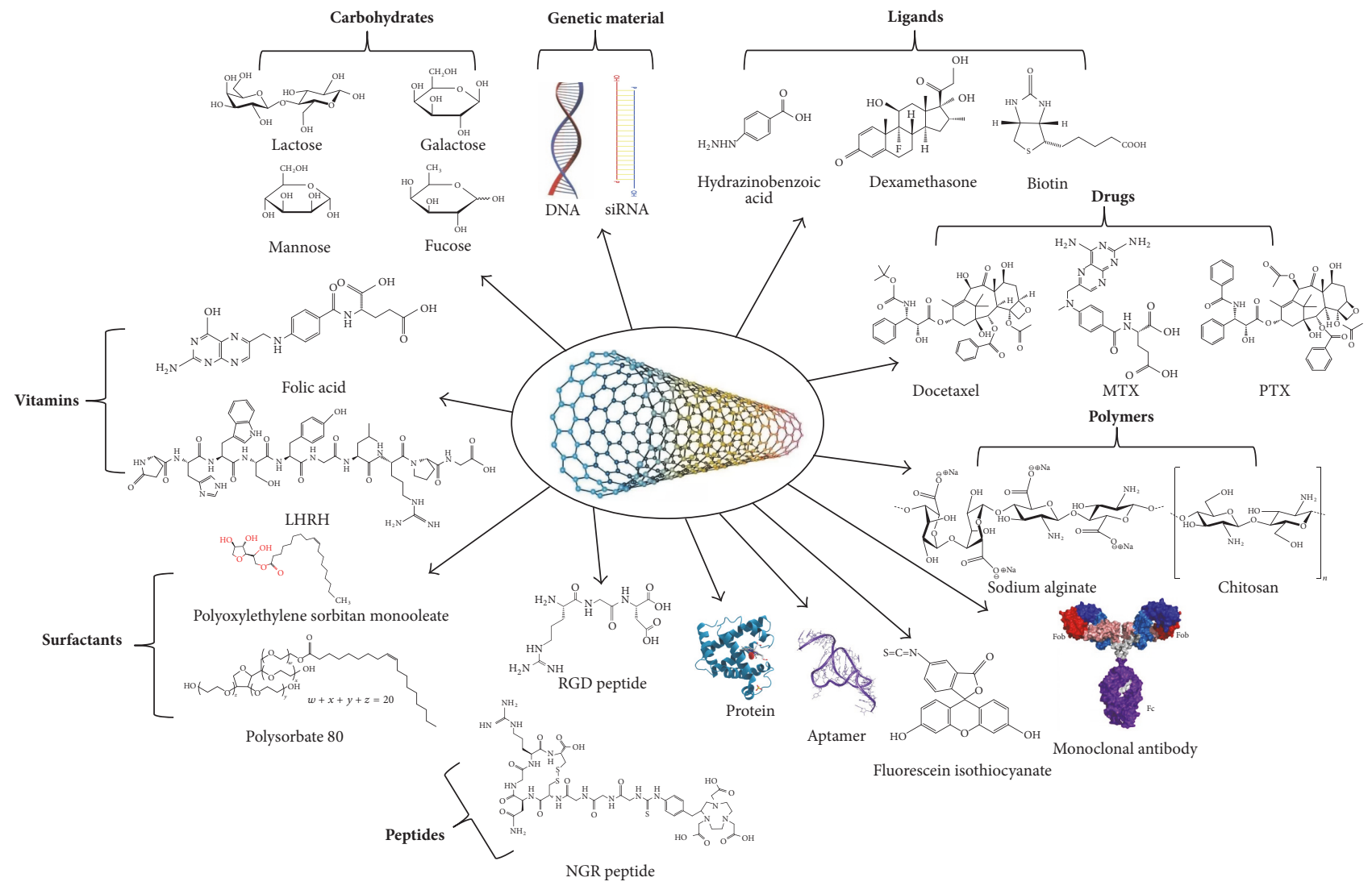

FIGURE 2: Examples of bioactive compounds conjugated through carbon nanotubes [31].

and oligomers can be bound to CNTs, which lead to the noncovalent functionalization of CNTs. The noncovalent functionalization of CNTs mainly related to the following several aspects, including pi stacking and Van der Waals and hydrophobic interactions. Hydrophobic or $\mathrm{p}-\mathrm{p}$ interactions are necessary for noncovalent stabilization. The covalent functionalization of CNTs can be completed via the "ends and defects" and "side walls" functionalization. The ends and defects technique has higher specificity and reaction than side walls functionalization [45]. The surface of pristine of CNTs can adsorb polymeric anticancer agents in a lot of cells. The interactions between the hydrophobic chains of the adsorbed molecules and the hydrophobic surface of CNTs can control the binding. In addition, p-p stacking interactions have also been considered to be related to such interactions [46]. The primary advantage of this kind of binding is to reduce the damage to the surface of CNTs to the minimal degree. According to some reports, the aromatic structure and electronic characteristics of CNTs can be preserved via noncovalent attachment. Due to chemical treatment, the emergence of charge on the surface of nanotube is able to adsorb the molecules via ionic interaction $[35,47,48]$. Some researchers have also been devoted to the debunching and solubilization of CNTs; they used nucleic acids and amphiphilic polymers on the basis of the p-p stacking interactions between the CNTs surface and aromatic bases/amino acids appearing in the structural backbone of these functional biomolecules. Meanwhile, the noncovalent bond provides a weak strength for CNTs. These make it unsuitable for tumortargeted drug delivery [49].

3.2. Covalent Functionalization. The covalent functionalization of CNTs can be achieved via the functionalization of "ends and defects" and "side walls." The ends and defects technology has high specificity and reaction compared with the function of side walls [45]. The covalent functionalization of CNTs also can be finished via the bond between the surfaces of CNTs and the biocompatible groups. Using this way, the surface of CNTs has been modified by different techniques, and this modification provides an appropriate platform on the surface of these materials. At the same time, it also makes covalent biocompatible groups integrate with CNTs. The surface oxidation treatment is a usually way of originating covalent bonding [35]. The surface of CNTs can be cut via treatment with strong acid solutions and then lead to carboxylic group being exposed at the defect point, predominately on the open ends. CNTs in concentrated sulphuric and nitric acid have been treated and heated by ultrasonic wave. The side wall covalent functionalization can be achieved via this process, and carboxylic acid group can attach to CNTs and then make CNTs become water-soluble. CNTs can be cut and oxidized to produce a certain number of carboxylic groups and then be subsequently derivatized with different types of molecules. CNTs can be oxidized by the 




Figure 3: Biomedical applications of functionalized CNTs.

combination between nitric and sulphuric acid. Meanwhile, the biocompatibility of CNTs can be enhanced via the presence of a carboxylic group. According to extra reactions, the sidewalls of CNTs have been directly functionalized. The introductions of moieties with no the exterior surface of the nanotube produces exclusion between the nanotubes; this behavior makes them easily dispersible into the solvent [22, 50-54].

The carboxyl groups at the surface of CNTs can lead to a highly negative charge, which enhance the hydrophilicity of these materials $[34,35]$. PEG is a hydrophilic substance, and it can cover the surface of CNTs and make oxidized CNTs more biostable [55]. The stable functionalized CNTs can be obtained via covalent coupling, which makes it become a more suitable tool for drug delivery. But during this process, the side wall of the CNTs has been damaged, which leads to the change of other properties of the CNTs $[49,56]$. So the functionalization of CNTs should not be used in some applications, for example, imaging. Further, the function of CNTs can promote their applications (Table 1) [57]. These

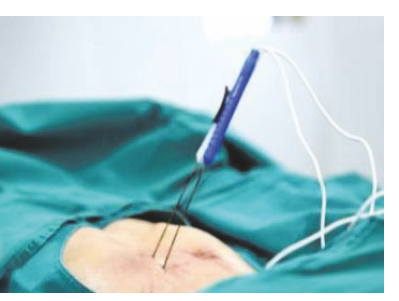

Minimally invasive surgery 





it is based on the biochemical and metabolic changes. Fluorodeoxyglucose (FDG) is one of the general radiopharmaceutical used in the medical imaging modality PET. It does not have the high specificity for cancer, but about $95 \%$ of the medical imaging technology is currently using it due to its promotion of metabolism [58-61]. It is urgent to find new methods for early cancer detection and diagnosis.

Some classic proteins are usually overexpressed in cancer cells, which can afford a chance for the early detection of the cancer. Clinically, several significant tumor markers have been widely used in the diagnosis of some cancers, including alpha-fetoprotein (AFP), carcinoma antigen 125 (CA125), carcinoembryonic antigen (CEA), human chorionic gonadotropin (hCG), carbohydrate antigen 19-9 (CA19-9), and prostate specific antigen (PSA). However, due to the low sensitivity and specificity for the early detection of cancer, these markers are only used in the following aspects, including deciding prognosis, predicting treatment responses, and keeping supervision for surgical treatment $[62,63]$. On the other hand, due to their unique electronic, mechanical, and thermal properties, CNTs have been suggested as a hopeful tool for detecting the expression of typical biological molecules at early stage of cancer. The novel methods based on the CNTs have been achieved, which have high sensitivity and wide linear and ultralow detection. Moreover, the new system of CNTs can save time compared with the conventional ELISA commercial test kits while achieving the similar selectivity [64]. More importantly, this approach can provide handheld equipment with less expense compared with currently methods, such as immunoassays, test-strips, and kits [65]. In summary, the CNTs-based detection may be a selective way for cancer diagnosis and treatment in clinical analysis in the near future.

The discovery of biomarkers that can be used in the diagnosis of cancer has become an emergent work in recent years. Due to radical resection of the tumor being the only method to treat this disease at the early stage, the early screening and detection have become very important for the therapy of cancer. At the early stage most cancers has no obvious symptoms, and the traditional clinical cancer imaging technologies do not have enough resolution for early diagnosis and assessment of prognosis. The change of tissue properties is the basis for the diagnosis of cancer at the early stages, including optical absorption, mechanical properties, and RF absorption. It is very important to find new techniques for the early detection of cancer. Here, we provide a new view about how nanotubes have been used in the different diagnostic principles.

4.1. Photoacoustic Imaging. As a new imaging technology recently, photoacoustic imaging (PAI) was generally used in the different biomedical fields [66-69]. The theory of PAI is simple. Pulse laser is assimilated via absorptiometric molecule in the biological sample to produce heat, which can induce short-lived thermoelastic inflation, thereby resulting in broadband ultrasonic emission, and an ultrasound microphone can explore all these alterations; subsequently these detected signals were used to construct $2 \mathrm{D}$ or $3 \mathrm{D}$ images [66]. The primary advantage of PAI is to provide higher spatial resolution and imaging of deeper tissues compared to the most optical imaging methods. The management of external photoacoustic contrast-mediums has become a necessary task. All kinds of nanomaterials have been applied as contrast-mediums in PAI, and these materials have strong capability to absorb light at near-infrared region (NIR) [66, 70-72]. Many contrast-mediums have been studied for PAI; however they cannot reach to the target site of disease in living subjects.

Due to MWNTs and SWNTs having strong NIR absorbance, they were used as a photothermal agent [73-75]. In addition, the property of firm NIR absorbancy makes NTs become perfect contrast-mediums for PAI. Some reports have demonstrated the application of SWNTs in PAI, including in vitro and in vivo [76, 77]. SWNTs can offer more than twofold signal amplification in thermoacoustic tomography (TAT) and more than sixfold signal enlargement in photoacoustic tomography (PAT) compared with control. Due to SWNTs being able to give maximum signal compared with other carbon materials, fullerenes, and graphitic microparticles, this characteristic makes them applicable in the PAI as ideal contrast-mediums [78].

When SWNTs have ability to keep their integrity, they will be combined with other contrast-mediums in order to increase the photoacoustic signal of SWNTs. SWNTs have to be combined with some gold-coated layers or bioorganic molecules to improve their absorbance in the NIR areas. Gold coating can increase the inherent photoacoustic signal of SWNTs, so some researchers have developed "golden nanotubes" (GNTs) [79]. Moreover, some NIR dyes such as indocyanine green (IGG) molecules [80] can be loaded on PEGylated SWNTs to improve the optical density, which result in the presence of multiplexed PAI probes [81].

Hence, CNTs that possess strong NIR absorbance have become a hopeful contrast agent in PAI. Other than the application of their innate optical absorbance, CNTs can also be combined with other absorptivity nanomaterials in order to enhance or multiplex PAI, which make CNTs also a universal nanoplatform. Now majority of CNTs-based PAI probes depended on SWNTs; MWNTs can also be helpful for this imaging technique.

4.2. Fluorescence Imaging. Fluorescence imaging (FI) performs important functions for medical diagnosis. But the penetration depth has limited their further application in fluorescence imaging [82]. In order to solve this problem, some researchers have been to continually advance and improve fluorescence probes. These probes can be excited and sent wavelengths reach to the biological transparent NIR window [83].

As synthesized SWNTs, only a small number can produce fluorescence after laser stimulation; meanwhile most of SWNTs have not generated fluorescence under the excitation of laser wavelength, and these no fluorescent SWNTs are dark field images. So once polarization-purified SWNTs are applied, most of nanotubes injected into animal can lead to significant decrease. Van Hoff singularities (VHSs) can provide a good character for the density of states (DOS), and VHSs have limited narrow energy bands with high DOS 
$[84,85]$. The energy band gap is in the order of $1 \mathrm{eV}$ between every semiconducting SWNTs, this character can ensure the fluorescence in the NIR-II region under the stimulation in the NIR-I region [85]. Furthermore, the large Stoke-shift between the excitation at $550-850 \mathrm{~nm}$ and emission at $900-1600 \mathrm{~nm}$ would dramatically lower the autofluorescence of biological tissues during imaging, and this condition can improve the sensitivity of imaging in vivo [86].

Robinson et al. have constructed a well functionalized SWNTs formulation; this formulation has a half-life of $30 \mathrm{~h}$ in blood circulation in vivo, thus leading to more than $\sim 30 \%$ inject dose (\% ID/g) drug accumulated [87]. High fluorescent video rate imaging and principle component analysis (PCA) have been applied to monitor the fluorescent signals in tumors and other organs at the first time. After $20 \mathrm{~s}$ injection they have seen the obvious fluorescent signals in tumor, and the fluorescence signal can be kept up to $72 \mathrm{~h}$. Furthermore, they have also seen the colocalization between tumor vasculatures and SWNTs in tumor inside via using technology of 3D reconstruction of NIR-II fluorescence signals. This discovery indicated that the effect of permeability and retention (EPR) may play vital role in mediating the tumor accumulation of nanotubes.

Hence, the SWNTs-based NIR-II FI has enormous potential in the field of biomedical diagnostics, and its peculiar advantages have been proved compared with other existing imaging technologies. Furthermore, different SWNTs have different excitation and emission wavelengths, and this feature makes them become potentially useful material for multicolor NIR-II fluorescence imaging. In other fluorescence enlargement technologies, such as based on surface resonance, the gold substrate can enhance the fluorescence of SWNT [88], and maybe these technologies can combine to further improve the sensitivity of imaging and detection.

4.3. Raman Image. Raman scattering is a photon scattering process, which refers to emission of photons with shifted wavelengths under light stimulation. The inherent Raman scattering signals of molecules do not involve enhancement mechanism such as surface enhanced Raman scattering (SERS) and are usually rather weak. SWNTs have various Raman peaks, which include the radical breathing mode (RBM, 100-300 $\mathrm{cm}^{-1}$ ) and tangential G band $\left(\sim 1580 \mathrm{~cm}^{-1}\right)$. These Raman peaks accord with the oscillation of carbon atoms in the radical direction and in the tangential direction, respectively. Because Raman spectroscopy can provide particular information on the chemical composition of cells and tissues, it has been studied in the application of biomedical diagnostics. It is very easy to distinguish the autofluorescence background due to the sharp and narrow Raman peaks of SWCNTs. The Raman excitation and scattering photons of SWCNTs can reach to the NIR region, and this is a transparent window for in vivo imaging. Dai and coworker have found various Raman imaging of live cells with isotopically modified SWCNTs [89]. In this work, SWCNTs with different isotope $\left({ }^{12} \mathrm{C}\right.$ and/or $\left.{ }^{13} \mathrm{C}\right)$ compositions (which have distinct Raman $\mathrm{G}$ band peaks) have been studied, while different cancer cells with different surface receptors were applied to receptor-specific targeting. Each targeting ligand can recognize its specific receptor, which led to the multicolor Raman imaging of cells in a multiplexed manner. When pure

${ }^{12} \mathrm{C}$ - and ${ }^{13} \mathrm{C}$-SWCNTs are conjugated by different antibodies, multiplexed Raman detection of different proteins can be proved.

\section{The Application of Carbon Nanotubes in Cancer Therapeutics}

At the early stage, the only effective method of cancer treatment is radical resection. In recent years, some methods for cancer therapy have appeared with the development of biotechnology; however, a more efficient method for specific target delivery of chemodrugs still needs to be developed $[90,91]$. In the past decades, various drug delivery systems were evaluated in order to deliver some chemicals to tumors, for example, doxorubicin (DOX) and paclitaxel (PTX). These delivery systems include liposome, natural/synthetic polymers, and nanoparticles. In these methods, liposomes and polymeric materials are widely used. Unfortunately, the inorganic materials, which have unique physiochemical properties, are largely ignored. CNTs have unique properties, which make them suitable carriers for target-specific drugloadable delivery. CNTs have specific advantages as drugcarriers, which include thermal conductivity, rigid structural properties capable of postchemical modification, sufficient surface-to-volume ratio, and excellent biocompatibility.

In addition, the drugs with water insolubility, antigens, antibodies, and nucleic acids can also be delivered into tumor cells via these carbon-based materials, and the unloading of therapeutic molecules is also very safe in tumor cells; then anticancer activity was efficiently enhanced. In this review, we have summarized the application of CNTs in cancer treatment from several aspects.

5.1. CNTs in Cancer Chemotherapy. Medicine for the treatment through a series of biologic obstacles is the first step, which is a vital link for the effect of chemotherapy. These obstacles include hepatic and renal clearance, hydrolysis, enzymolysis, endocytosis, and lysosomal degradation. Some chemotherapeutic drugs have poor solubility, low stability, and high toxicity for normal cells and tissues, which have a serious influence on their efficiency. However, CNTs-based carriers can enhance the biodistribution and prolong blood circulation of therapeutics; then the pharmaceutical efficacy can be increased and the usage dose can be decreased. In view of the fact that the biocompatible SWNT, Liu et al. [92], made DOX loaded onto branched PEG-functionalized SWNTs designed in order to prolong blood circulation time, they have injected the SWNT-DOX complex to tumor mice. They found that DOX can be delivered into tumors, and SWNT can be cleared from systemic blood circulation via renal excretion. However, physical loading for PTX is very difficult due to its poor solubility in aqueous solution. In order to overcome this hurdle, Lay et al. produced both PEG-graft single-walled CNTs (PEG-gSWNTs) and PEG-graft multiwalled CNTs (PEG-gMWNTs) to enhance loading ability [93]. They found that the delivery of PTX can be sustained over 40 days in vitro. With the development of technology, some researchers 


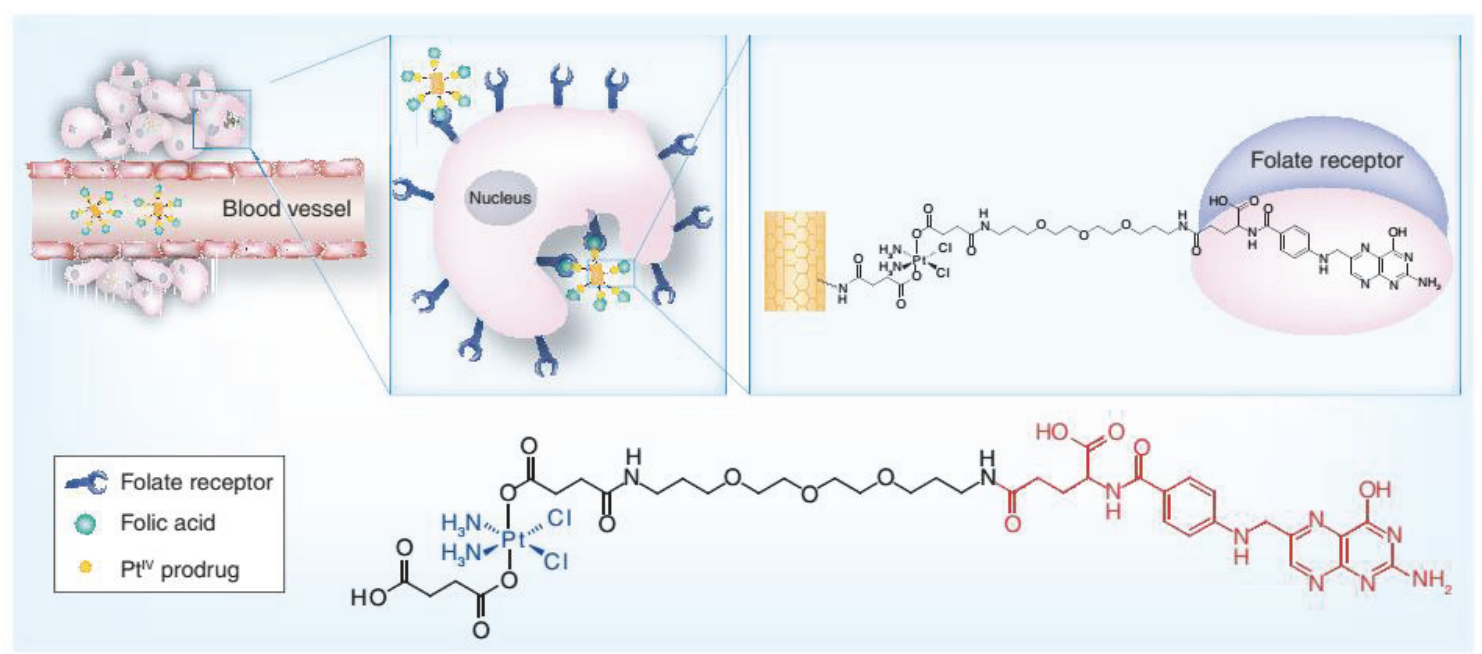

FIGURE 4: Folate-mediated cancer targeting using single-walled carbon nanotubes conjugated with platinum-containing anticancer drug and its subsequent endocytosis [27].

have modified SWNTs carrier to improve their efficiency of delivery, such as the epidermal growth factor- (EGF-) mediated SWNT delivery of an anticancer agent cisplatin [94]. In addition, MWNTs can be used for thermal ablation as a carbon-based nanomaterial, which results in hyperthermia for destroying cancer cells.

5.2. CNTs in Drug Delivery. The systemic toxicity of chemotherapeutic agents is often neglected due to the lack of selectivity conventional administration. Furthermore, these agents have limited solubility and inability to pass through cellular barriers [95], especially the clinical routines which are lacking for overcoming multidrug resistant (MDR) cancer [96]. In recent years, these problems have been studied by researchers all over the world. Several novel targeted therapies have been discovered, which are based on CNTs materials. These delivery systems usually include three portions, and they are functional CNTs, targeted ligands, and anticarcinogen. Many drugs, polypeptides and nucleonic acid, can be integrated into CNTs because of their unique ultrahigh surface area. Due to the existence of endocytosis and other mechanisms, the functional CNTs can pass through the membrane of mammalian cells [94, 97, 98]. They have the unique ability to identify the specific surface receptors when drug delivery systems (NDDs) interact with cancer cells, which can result in receptor-mediated endocytosis. Anticancer drugs can effectively reach the cells via the CNTsbased delivery system, and these advantages make CNTs become ideal candidates for drug delivery. The cancer cells can effectively and specifically absorb the CNTs complex, and the chemotherapeutic agents can be released into intracellular space; then these drugs can more effectively curb the spread of cancer cells than uncontrolled drugs. In addition, the cytotoxicity was decreased by using this novel approach, and the severe side-effects could be avoided [94]. SWNTs can offer higher ability of drug loading compared with the traditional liposomes and dendrimer drug carries due to their extremely high specific surface area [49]. Figure 4 indicates a delivery system that SWCNTs medicated, which can deliver drugs for treatment of cancer. Overall, these advantages clearly indicated that CNTs can be used for targeted delivery system in the future.

5.3. Photothermal Therapy. While thermotherapy has been applied in treatment of tumor patients for long time [99], photothermal therapy is still considered to be one of the best methods to treat cancer. Recently, some researchers have been reported indicating the possibility of heating CNTs injected into cancer cells and thus induce their death. Photothermal therapy as a noninvasive, uninjurious and effective therapeutic technology can be heated locally just to the tumor tissues via NIR, and then tumor cells have been destroyed $[55,100,101]$. In addition, the side-effects on other parts can be decreased to the minimum by using this method [102-106]. The CNTs have good light absorption performance under NIR and radio-frequency radiation [107, 108]. Furthermore, the length of nanotubes is a very vital element, and it decides the capacity of heat transmission and cancer cell erosion [109]. The CNTs-based photothermal therapy systems can combine with chemotherapy and gene treatment techniques to obtain more efficiency for treating cancer $[55,110]$.

To enhance the selectivity in the treatment of cancer and to reduce the side-effects to normal cells, the targeted CNTs can be achieved via covalent or noncovalent coating with cell-bound ligands, for example, monoclonal antibodies (MAbs) or peptides $[55,104,110,111]$. In view of photothermal transduction influence, nanotubes have been supposed and developed as photothermal agents for killing cancer, and this function can be achieved via heating the nanotube to $50-70^{\circ} \mathrm{C}$ through sustaining laser irradiation at high power density $\left(3.5-35 \mathrm{~W} / \mathrm{cm}^{2}\right)$ for a long time (3-4 $\left.\mathrm{min}\right)$ [112]. Furthermore, photothermal therapy is based on absorption properties of antibody-conjugated nanomaterials, and this therapy method 
has been confirmed having selective ability to kill tumor cells, but the healthy cells will not be affected [113-115].

5.4. Delivery of Immunotherapeutics. The antitumor immunotherapy with CNTs-based has been studied. Tumor cell vaccines (TCV) were used in this method and the immune response of the patient against the tumor itself was triggered by inactivated cancer cells or dendritic cells presenting tumor antigens [116]. In order to enhance the efficacy of TCV, the oxidized MWCNTs can be covalently coupled with tumor lysate proteins by an amide bond. On the other hand, Villa et al. have developed the capacity of SWCNTs to act as antigen presenting carries to increase the response to weak immunogenic peptides [117]. The Wilms tumor protein (WT1) was covalently conjugated with SWCNTs, and this modification makes it become a vaccine target for many human cancers. In their study, SWCNTs-WT1 can be rapidly uptaken by APC in vitro, which is also dependent of dose. In addition, they used SWCNTs-WT1 adjuvant to immune mice and found that mice can cause a specific humoral immune reaction, and they do not see other response against the peptide alone against the peptide mixed with the adjuvant. These results suggested that SWCNTs can be a new tool to deliver poorly immunogenic peptides to the immune system, thus enhancing the efficiency of vaccine treatment.

\section{Conclusion}

In recent years, nanotechnology has dramatically drawn attention and interest in medicine and biology. Carbon nanotubes are one of the nanomaterials, which include two forms. CNTs have been functionalized via covalent and noncovalent interaction. Functionalized CNTs can be used as carriers to play their roles in delivery system. The research of their biomedical applications just has been less than twenty years. The CNTs have better biocompatibility and multimodal functions after their surfaces were modified; therefore the therapeutic effect was better than before, especially for the treatment of cancer. Carriers CNTs have many advantages, including high load of drugs, good penetrability, photothermal ablation, and inherent diagnostic capability. These advantages are the key elements for making them as appropriate nanocarriers in tumor treatment. CNTs have the ability to deliver biologically active molecules to cytoplasm, and they need to bypass many biological barriers and play as a cellular needle during this process. Furthermore, CNTs have more surface areas and internal antrums, which can be loaded with targeted ligands and filled with diagnostic or therapeutic agents. In addition, CNTs also have unparalleled electrical and thermal conductivity characters. These advantages make their clinical application possible. Despite CNTs having great promise for the treatment of cancer, we cannot neglect their nonbiodegradable nature and cytotoxicity in clinical use. The emergence of new techniques for surface functionalization may help reduce these disadvantages. We believe that this novel CNT based platform can provide us with a safer and more effective way for the cancer therapy in the near future.

\section{Conflicts of Interest}

The authors declare that they have no conflicts of interest.

\section{Authors' Contributions}

Zhou Chen, Aili Zhang, and Xiaobing Wang contributed equally to this work.

\section{Acknowledgments}

This work is supported by the Priority Academic Program Development of Jiangsu Higher Education Institutions.

\section{References}

[1] S. Y. Madani, F. Shabani, M. V. Dwek, and A. M. Seifalian, "Conjugation of quantum dots on carbon nanotubes for medical diagnosis and treatment," International Journal of Nanomedicine, vol. 8, pp. 941-950, 2013.

[2] Z. Yang, L. Chang, C.-L. Chiang, and L. J. Lee, "Micro-/nanoelectroporation for active gene delivery," Current Pharmaceutical Design, vol. 21, no. 42, pp. 6081-6088, 2015.

[3] Z. Yang, B. Yu, J. Zhu et al., "A microfluidic method to synthesize transferrin-lipid nanoparticles loaded with siRNA LOR-1284 for therapy of acute myeloid leukemia," Nanoscale, vol. 6, no. 16, pp. 9742-9751, 2014.

[4] C. Zhou, Z. Yang, and L. Teng, "Nanomedicine based on nucleic acids: pharmacokinetic and pharmacodynamic perspectives," Current Pharmaceutical Biotechnology, vol. 15, no. 9, pp. 829838,2015

[5] X. Yang, S. Yang, H. Chai et al., "A novel isoquinoline derivative anticancer agent and its targeted delivery to tumor cells using transferrin-conjugated liposomes," PLoS ONE, vol. 10, no. 8, Article ID e0136649., 2015.

[6] B. Yu, X. Wang, C. Zhou et al., "Insight into mechanisms of cellular uptake of lipid nanoparticles and intracellular release of small RNAs," Pharmaceutical Research, vol. 31, no. 10, pp. 26852695, 2014

[7] J. Xie, L. Teng, Z. Yang et al., "A polyethylenimine-linoleic acid conjugate for antisense oligonucleotide delivery," BioMed Research International, vol. 2013, Article ID 710502, 7 pages, 2013.

[8] J. Xie, Z. Yang, C. Zhou, J. Zhu, R. J. Lee, and L. Teng, "Nanotechnology for the delivery of phytochemicals in cancer therapy," Biotechnology Advances, vol. 34, no. 4, pp. 343-353, 2016.

[9] Z. Chen, A. Zhang, J. Hu, X. Wang, and Z. Yang, "Electrospun nanofibers for cancer diagnosis and therapy," Biomaterials Science, vol. 4, no. 6, pp. 922-932, 2016.

[10] Z. Chen, M. Cong, J. Hu, Z. Yang, and Z. Chen, "Preparation of functionalized $\mathrm{TiO}_{2}$ nanotube arrays and their applications," Science of Advanced Materials, vol. 8, no. 6, pp. 1231-1241, 2016.

[11] Z. Yang, J. Xie, J. Zhu et al., "Functional exosome-mimic for delivery of siRNA to cancer: in vitro and in vivo evaluation," Journal of Controlled Release, vol. 243, pp. 160-171, 2016.

[12] L. Sha, Z. Chen, Z. Chen, A. Zhang, and Z. Yang, "Polylactic acid based nanocomposites: Promising safe and biodegradable materials in biomedical field," International Journal of Polymer Science, vol. 2016, Article ID 6869154, 11 pages, 2016. 
[13] C. Kang, Y. Sun, J. Zhu et al., "Delivery of nanoparticles for treatment of brain tumor," Current Drug Metabolism, vol. 17, no. 8, pp. 745-754, 2016.

[14] Z. Chena, A. Zhang, Z. Yang et al., "Application of DODMA and derivatives in cationic nanocarriers for gene delivery," Current Organic Chemistry, vol. 20, no. 17, pp. 1813-1819, 2016.

[15] L. J. Lee, Z. Yang, M. Rahman et al., "Extracellular mRNA detected by tethered lipoplex nanoparticle biochip for lung adenocarcinoma detection," American Journal of Respiratory \& Critical Care Medicine, vol. 193, no. 12, pp. 1431-1433, 2016.

[16] N. G. Sahoo, H. Bao, Y. Pan et al., "Functionalized carbon nanomaterials as nanocarriers for loading and delivery of a poorly water-soluble anticancer drug: a comparative study," Chemical Communications, vol. 47, no. 18, pp. 5235-5237, 2011.

[17] P. Utreja, S. Jain, and A. K. Tiwary, "Novel drug delivery systems for sustained and targeted delivery of anti-cancer drugs: current status and future prospects," Current Drug Delivery, vol. 7, no. 2, pp. 152-161, 2010.

[18] K. Kostarelos, L. Lacerda, G. Pastorin et al., "Cellular uptake of functionalized carbon nanotubes is independent of functional group and cell type," Nature Nanotechnology, vol. 2, no. 2, pp. 108-113, 2007.

[19] N. W. Kam, Z. Liu, and H. Dai, "Carbon nanotubes as intracellular transporters for proteins and DNA: an investigation of the uptake mechanism and pathway," Angewandte Chemie, vol. 118, no. 4, pp. 591-595, 2006.

[20] C. Fabbro, H. Ali-Boucetta, T. D. Ros, K. Kostarelos, A. Bianco, and M. Prato, "Targeting carbon nanotubes against cancer," Chemical Communications, vol. 48, no. 33, pp. 3911-3926, 2012.

[21] R. Singh, D. Pantarotto, L. Lacerda et al., "Tissue biodistribution and blood clearance rates of intravenously administered carbon nanotube radiotracers," Proceedings of the National Academy of Sciences of the United States of America, vol. 103, no. 9, pp. 33573362, 2006.

[22] L. Lacerda, H. Ali-Boucetta, M. A. Herrero et al., "Tissue histology and physiology following intravenous administration of different types of functionalized multiwalled carbon nanotubes," Nanomedicine, vol. 3, no. 2, pp. 149-161, 2008.

[23] L. Lacerda, M. A. Herrero, K. Venner, A. Bianco, M. Prato, and K. Kostarelos, "Carbon-nanotube shape and individualization critical for renal excretion," Small, vol. 4, no. 8, pp. 1130-1132, 2008.

[24] M. R. McDevitt, D. Chattopadhyay, J. S. Jaggi et al., "PET imaging of soluble yttrium-86-labeled carbon nanotubes in mice," PLoS ONE, vol. 2, no. 9, article no. e907, 2007.

[25] Z. Liu, C. Davis, W. Cai, L. He, X. Chen, and H. Dai, "Circulation and long-term fate of functionalized, biocompatible single-walled carbon nanotubes in mice probed by Raman spectroscopy," Proceedings of the National Academy of Sciences of the United States of America, vol. 105, no. 5, pp. 1410-1415, 2008.

[26] K. Kostarelos, A. Bianco, and M. Prato, "Promises, facts and challenges for carbon nanotubes in imaging and therapeutics," Nature Nanotechnology, vol. 4, no. 10, pp. 627-633, 2009.

[27] A. Ruggiero, C. H. Villa, E. Bander et al., "Paradoxical glomerular filtration of carbon nanotubes," Proceedings of the National Academy of Sciences of the United States of America, vol. 107, no. 27, pp. 12369-12374, 2010.

[28] P. C. B. D. Faria, L. I. D. Santos, J. P. Coelho et al., "Oxidized multiwalled carbon nanotubes as antigen delivery system to promote superior CD8+ T Cell response and protection against Cancer," Nano Letters, vol. 14, no. 9, pp. 5458-5470, 2014.
[29] A. Jemal, S. S. Devesa, P. Hartge, and M. A. Tucker, "Recent trends in cutaneous melanoma incidence among whites in the United States," Journal of the National Cancer Institute, vol. 93, no. 9, pp. 678-683, 2001.

[30] Y. Yan, M. B. Chan-Park, and Q. Zhang, "Advances in carbonnanotube assembly," Small, vol. 3, no. 1, pp. 24-42, 2007.

[31] P. Kesharwani, V. Mishra, and N. K. Jain, "Validating the anticancer potential of carbon nanotube-based therapeutics through cell line testing," Drug Discovery Today, vol. 20, no. 9, pp. 1049-1060, 2015.

[32] D. S. Bethune, C. H. Kiang, M. S. de Vries et al., "Cobaltcatalysed growth of carbon nanotubes with single-atomic-layer walls," Nature, vol. 363, no. 6430, pp. 605-607, 1993.

[33] N. K. Mehra, V. Mishra, and N. K. Jain, "A review of ligand tethered surface engineered carbon nanotubes," Biomaterials, vol. 35, no. 4, pp. 1267-1283, 2014.

[34] E. Bekyarova, Y. Ni, E. B. Malarkey et al., "Applications of Carbon Nanotubes in Biotechnology and Biomedicine," Journal of Biomedical Nanotechnology, vol. 1, no. 1, pp. 3-17, 2005.

[35] C. Klumpp, K. Kostarelos, M. Prato, and A. Bianco, "Functionalized carbon nanotubes as emerging nanovectors for the delivery of therapeutics," Biochimica et Biophysica Acta-Biomembranes, vol. 1758, no. 3, pp. 404-412, 2006.

[36] S. Y. Madani, N. Naderi, O. Dissanayake, A. Tan, and A. M. Seifalian, "A new era of cancer treatment: carbon nanotubes as drug delivery tools," International Journal of Nanomedicine, vol. 6, pp. 2963-2979, 2011.

[37] A. Bianco, K. Kostarelos, and M. Prato, "Applications of carbon nanotubes in drug delivery," Current Opinion in Chemical Biology, vol. 9, no. 6, pp. 674-679, 2005.

[38] J. Liu, A. G. Rinzler, H. Dai et al., "Fullerene pipes," Science, vol. 280, no. 5367, pp. 1253-1256, 1998.

[39] D. Tasis, N. Tagmatarchis, V. Georgakilas, and M. Prato, "Soluble carbon nanotubes," Chemistry - A European Journal, vol. 9, no. 17, pp. 4000-4008, 2003.

[40] V. Georgakilas, K. Kordatos, M. Prato, D. M. Guldi, M. Holzinger, and A. Hirsch, "Organic functionalization of carbon nanotubes," Journal of the American Chemical Society, vol. 124, no. 5, pp. 760-761, 2002.

[41] Z. Chen, W. Wu, Z. Chen, X. Cong, and J. Qiu, "Microstructural characterization on $\mathrm{ZrC}$ doped carbon/carbon composites," Ceramics International, vol. 38, no. 1, pp. 761-767, 2012.

[42] A. Bianco, K. Kostarelos, C. D. Partidos, and M. Prato, "Biomedical applications of functionalised carbon nanotubes," Chemical Communications, no. 5, pp. 571-577, 2005.

[43] P. Kesharwani, R. Ghanghoria, and N. K. Jain, "Carbon nanotube exploration in cancer cell lines," Drug Discovery Today, vol. 17, no. 17-18, pp. 1023-1030, 2012.

[44] I. D. Rosca, F. Watari, M. Uo, and T. Akasaka, "Oxidation of multiwalled carbon nanotubes by nitric acid," Carbon, vol. 43, no. 15, pp. 3124-3131, 2005.

[45] S. Vardharajula, S. Z. Ali, P. M. Tiwari et al., "Functionalized carbon nanotubes: biomedical applications," International Journal of Nanomedicine, vol. 7, pp. 5361-5374, 2012.

[46] V. S. Thakare, M. Das, A. K. Jain, S. Patil, and S. Jain, "Carbon nanotubes in cancer theragnosis," Nanomedicine, vol. 5, no. 8, pp. 1277-1301, 2010.

[47] R. M. Lucente-Schultz, V. C. Moore, A. D. Leonard et al., "Antioxidant single-walled carbon nanotubes," Journal of the American Chemical Society, vol. 131, no. 11, pp. 3934-3941, 2009. 
[48] N. Jia, Q. Lian, H. Shen, C. Wang, X. Li, and Z. Yang, "Intracellular delivery of quantum dots tagged antisense oligodeoxynucleotides by functionalized multiwalled carbon nanotubes," Nano Letters, vol. 7, no. 10, pp. 2976-2980, 2007.

[49] Z. Liu, X. Sun, N. Nakayama-Ratchford, and H. Dai, "Supramolecular chemistry on water-soluble carbon nanotubes for drug loading and delivery," ACS Nano, vol. 1, no. 1, pp. 50-56, 2007.

[50] A. Hirsch, "Functionalization of single-walled carbon nanotubes," Angewandte Chemie - International Edition, vol. 41, no. 11, pp. 1853-1859, 2002.

[51] G. Pastorin, W. Wu, S. Wieckowski et al., "Double functionalisation of carbon nanotubes for multimodal drug delivery," Chemical Communications, no. 11, pp. 1182-1184, 2006.

[52] Y.-J. Lu, K.-C. Wei, C.-C. M. Ma, S.-Y. Yang, and J.-P. Chen, "Dual targeted delivery of doxorubicin to cancer cells using folate-conjugated magnetic multi-walled carbon nanotubes," Colloids and Surfaces B: Biointerfaces, vol. 89, no. 1, pp. 1-9, 2012.

[53] N. Karousis, N. Tagmatarchis, and D. Tasis, "Current progress on the chemical modification of carbon nanotubes," Chemical Reviews, vol. 110, no. 9, pp. 5366-5397, 2010.

[54] V. Mishra, P. Kesharwani, and N. K. Jain, "SiRNA nanotherapeutics: a Trojan horse approach against HIV," Drug Discovery Today, vol. 19, no. 12, pp. 1913-1920, 2014.

[55] N. W. S. Kam, M. O'Connell, J. A. Wisdom, and H. Dai, “Carbon nanotubes as multifunctional biological transporters and nearinfrared agents for selective cancer cell destruction," Proceedings of the National Academy of Sciences of the United States of America, vol. 102, no. 33, pp. 11600-11605, 2005.

[56] S. Niyogi, M. A. Hamon, H. Hu et al., "Chemistry of singlewalled carbon nanotubes," Accounts of Chemical Research, vol. 35, no. 12, pp. 1105-1113, 2002.

[57] R. V. Mundra, X. Wu, J. Sauer, J. S. Dordick, and R. S. Kane, "Nanotubes in biological applications," Current Opinion in Biotechnology, vol. 28, pp. 25-32, 2014.

[58] S.-R. Ji, C. Liu, B. Zhang et al., "Carbon nanotubes in cancer diagnosis and therapy," Biochimica et Biophysica Acta-Reviews on Cancer, vol. 1806, no. 1, pp. 29-35, 2010.

[59] R. P. Levy, "PET-CT: evolving role in hadron therapy," Nuclear Instruments and Methods in Physics Research, Section B: Beam Interactions with Materials and Atoms, vol. 261, no. 1-2, pp. 782785, 2007.

[60] D. Papathanassiou, C. Bruna-Muraille, J.-C. Liehn, T. D. Nguyen, and H. Curé, "Positron emission tomography in oncology: present and future of PET and PET/CT" Critical Reviews in Oncology/Hematology, vol. 72, no. 3, pp. 239-254, 2009.

[61] D. Delbeke, H. Schöder, W. H. Martin, and R. L. Wahl, "Hybrid imaging (SPECT/CT and PET/CT): improving therapeutic decisions," Seminars in Nuclear Medicine, vol. 39, no. 5, pp. 308340, 2009.

[62] M. J. Duffy, "Role of tumor markers in patients with solid cancers: a critical review," European Journal of Internal Medicine, vol. 18, no. 3, pp. 175-184, 2007.

[63] L. K. Rogers, M. Robbins, D. Dakhlallah et al., "Attenuation of MIR-17 92 cluster in bronchopulmonary dysplasia," Annals of the American Thoracic Society, vol. 12, no. 10, pp. 1506-1513, 2015.

[64] N. V. Panini, G. A. Messina, E. Salinas, H. Fernández, and J. Raba, "Integrated microfluidic systems with an immunosensor modified with carbon nanotubes for detection of prostate specific antigen (PSA) in human serum samples," Biosensors and Bioelectronics, vol. 23, no. 7, pp. 1145-1151, 2008.
[65] J.-A. A. Ho, Y.-C. Lin, L.-S. Wang, K.-C. Hwang, and P.-T. Chou, "Carbon nanoparticle-enhanced immunoelectrochemical detection for protein tumor marker with cadmium sulfide biotracers," Analytical Chemistry, vol. 81, no. 4, pp. 1340-1346, 2009.

[66] H. Gong, R. Peng, and Z. Liu, "Carbon nanotubes for biomedical imaging: the recent advances," Advanced Drug Delivery Reviews, vol. 65, no. 15, pp. 1951-1963, 2013.

[67] X. Wang, Y. Pang, G. Ku, X. Xie, G. Stoica, and L. V. Wang, "Noninvasive laser-induced photoacoustic tomography for structural and functional in vivo imaging of the brain," Nature Biotechnology, vol. 21, no. 7, pp. 803-806, 2003.

[68] G. Ku and L. V. Wang, "Deeply penetrating photoacoustic tomography in biological tissues enhanced with an optical contrast agent," Optics Letters, vol. 30, no. 5, pp. 507-509, 2005.

[69] C. G. A. Hoelen, F. F. M. De Mul, R. Pongers, and A. Dekker, "Three-dimensional photoacoustic imaging of blood vessels in tissue," Optics Letters, vol. 23, no. 8, pp. 648-650, 1998.

[70] K. Yang, L. Hu, X. Ma et al., "Multimodal imaging guided photothermal therapy using functionalized graphene nanosheets anchored with magnetic nanoparticles," Advanced Materials, vol. 24, no. 14, pp. 1868-1872, 2012.

[71] G. Ku, M. Zhou, S. Song, Q. Huang, J. Hazle, and C. Li, “Copper sulfide nanoparticles as a new class of photoacoustic contrast agent for deep tissue imaging at $1064 \mathrm{~nm}$," ACS Nano, vol. 6, no. 8, pp. 7489-7496, 2012.

[72] A. Agarwal, S. W. Huang, M. O’Donnell et al., “Targeted gold nanorod contrast agent for prostate cancer detection by photoacoustic imaging," Journal of Applied Physics, vol. 102, no. 6, Article ID 064701, 2007.

[73] J. T. Robinson, K. Welsher, S. M. Tabakman et al., "High performance in vivo near-IR $(>1 \mu \mathrm{m})$ imaging and photothermal cancer therapy with carbon nanotubes," Nano Research, vol. 3, no. 11, pp. 779-793, 2010.

[74] X. Liu, H. Tao, K. Yang, S. Zhang, S.-T. Lee, and Z. Liu, "Optimization of surface chemistry on single-walled carbon nanotubes for in vivo photothermal ablation of tumors," Biomaterials, vol. 32, no. 1, pp. 144-151, 2011.

[75] H. K. Moon, S. H. Lee, and H. C. Choi, "In vivo near-infrared mediated tumor destruction by photothermal effect of carbon nanotubes," ACS Nano, vol. 3, no. 11, pp. 3707-3713, 2009.

[76] P. K. Avti, S. Hu, C. Favazza et al., "Detection, mapping, and quantification of single walled carbon nanotubes in histological specimens with photoacoustic microscopy," PLOS ONE, vol. 7, no. 4, Article ID e35064, 2012.

[77] L. Wu, X. Cai, K. Nelson et al., "A green synthesis of carbon nanoparticles from honey and their use in real-time photoacoustic imaging," Nano Research, vol. 6, no. 5, pp. 312-325, 2013.

[78] M. Pramanik, M. Swierczewska, D. Green, B. Sitharaman, and L. V. Wang, "Single-walled carbon nanotubes as a multimodalthermoacoustic and photoacoustic-contrast agent," Journal of Biomedical Optics, vol. 14, no. 3, Article ID 034018, 2009.

[79] J.-W. Kim, E. I. Galanzha, E. V. Shashkov, H.-M. Moon, and V. P. Zharov, "Golden carbon nanotubes as multimodal photoacoustic and photothermal high-contrast molecular agents," Nature Nanotechnology, vol. 4, no. 10, pp. 688-694, 2009.

[80] A. D. L. Zerda, Z. Liu, S. Bodapati et al., "Ultrahigh sensitivity carbon nanotube agents for photoacoustic molecular imaging in living mice," Nano Letters, vol. 10, no. 6, pp. 2168-2172, 2010.

[81] A. De La Zerda, S. Bodapati, R. Teed et al., "Family of enhanced photoacoustic imaging agents for high-sensitivity 
and multiplexing studies in living mice," ACS Nano, vol. 6, no. 6, pp. 4694-4701, 2012.

[82] X. He, J. Gao, S. S. Gambhir, and Z. Cheng, "Near-infrared fluorescent nanoprobes for cancer molecular imaging: status and challenges," Trends in Molecular Medicine, vol. 16, no. 12, pp. 574-583, 2010.

[83] B. Chance, "Near-infrared images using continuous, phasemodulated, and pulsed light with quantitation of blood and blood oxygenation," Annals of the New York Academy of Sciences, vol. 838, pp. 29-45, 1998.

[84] H. Kuzmany, B. Burger, M. Fally, A. G. Rinzler, and R. E. Smalley, "Effect of dimensionality in polymeric fullerenes and single-wall nanotubes," Physica B: Condensed Matter, vol. 244, pp. 186-191, 1998.

[85] A. Jorio, M. A. Pimenta, A. G. S. Filho, R. Saito, G. Dresselhaus, and M. S. Dresselhaus, "Characterizing carbon nanotube samples with resonance Raman scattering," New Journal of Physics, vol. 5, article 139, 2003.

[86] G. Hong, J. C. Lee, J. T. Robinson et al., "Multifunctional in vivo vascular imaging using near-infrared II fluorescence," Nature Medicine, vol. 18, no. 12, pp. 1841-1846, 2012.

[87] J. T. Robinson, G. Hong, Y. Liang, B. Zhang, O. K. Yaghi, and H. Dai, "In vivo fluorescence imaging in the second near-infrared window with long circulating carbon nanotubes capable of ultrahigh tumor uptake," Journal of the American Chemical Society, vol. 134, no. 25, pp. 10664-10669, 2012.

[88] G. Hong, S. M. Tabakman, K. Welsher, H. Wang, X. Wang, and H. Dai, "Metal-enhanced fluorescence of carbon nanotubes," Journal of the American Chemical Society, vol. 132, no. 45, pp. 15920-15923, 2010.

[89] Z. Liu, X. Li, S. M. Tabakman, K. Jiang, S. Fan, and H. Dai, "Multiplexed multicolor raman imaging of live cells with isotopically modified single walled carbon nanotubes," Journal of the American Chemical Society, vol. 130, no. 41, pp. 1354013541, 2008.

[90] B. Haley and E. Frenkel, "Nanoparticles for drug delivery in cancer treatment," Urologic Oncology: Seminars and Original Investigations, vol. 26, no. 1, pp. 57-64, 2008.

[91] D. M. Webster, P. Sundaram, and M. E. Byrne, "Injectable nanomaterials for drug delivery: carriers, targeting moieties, and therapeutics," European Journal of Pharmaceutics and Biopharmaceutics, vol. 84, no. 1, pp. 1-20, 2013.

[92] Z. Liu, A. C. Fan, K. Rakhra et al., "Supramolecular stacking of doxorubicin on carbon nanotubes for in vivo cancer therapy," Angewandte Chemie, vol. 48, no. 41, pp. 7668-7672, 2009.

[93] C. L. Lay, H. Q. Liu, H. R. Tan, and Y. Liu, "Delivery of paclitaxel by physically loading onto poly(ethylene glycol) (PEG)-graftcarbon nanotubes for potent cancer therapeutics," Nanotechnology, vol. 21, no. 6, Article ID 065101, 2010.

[94] A. A. Bhirde, V. Patel, J. Gavard et al., “Targeted killing of cancer cells in vivo and in vitro with EGF-directed carbon nanotubebased drug delivery," ACS Nano, vol. 3, no. 2, pp. 307-316, 2009.

[95] E. Heister, V. Neves, C. Tîlmaciu et al., “Triple functionalisation of single-walled carbon nanotubes with doxorubicin, a monoclonal antibody, and a fluorescent marker for targeted cancer therapy," Carbon, vol. 47, no. 9, pp. 2152-2160, 2009.

[96] L. S. Jabr-Milane, L. E. van Vlerken, S. Yadav, and M. M. Amiji, "Multi-functional nanocarriers to overcome tumor drug resistance," Cancer Treatment Reviews, vol. 34, no. 7, pp. 592602, 2008.
[97] N. Sinha and J. T.-W. Yeow, "Carbon nanotubes for biomedical applications," IEEE Transactions on Nanobioscience, vol. 4, no. 2, pp. 180-195, 2005.

[98] X. Zhang, L. Meng, Q. Lu, Z. Fei, and P. J. Dyson, “Targeted delivery and controlled release of doxorubicin to cancer cells using modified single wall carbon nanotubes," Biomaterials, vol. 30, no. 30, pp. 6041-6047, 2009.

[99] S. B. Field and N. M. Bleehen, "Hyperthermia in the treatment of cancer," Cancer Treatment Reviews, vol. 6, no. 2, pp. 63-94, 1979.

[100] X. Huang, I. H. El-Sayed, W. Qian, and M. A. El-Sayed, "Cancer cell imaging and photothermal therapy in the nearinfrared region by using gold nanorods," Journal of the American Chemical Society, vol. 128, no. 6, pp. 2115-2120, 2006.

[101] A. M. Gobin, M. H. Lee, N. J. Halas, W. D. James, R. A. Drezek, and J. L. West, "Near-infrared resonant nanoshells for combined optical imaging and photothermal cancer therapy," Nano Letters, vol. 7, no. 7, pp. 1929-1934, 2007.

[102] Z. Liu and R. Peng, "Inorganic nanomaterials for tumor angiogenesis imaging," European Journal of Nuclear Medicine and Molecular Imaging, vol. 37, no. 1, pp. S147-S163, 2010.

[103] S. Ghosh, S. Dutta, E. Gomes et al., "Increased heating efficiency and selective thermal ablation of malignant tissue with DNAencased multiwalled carbon nanotubes," ACS Nano, vol. 3, no. 9, pp. 2667-2673, 2009.

[104] R. Marches, P. Chakravarty, I. H. Musselman et al., "Specific thermal ablation of tumor cells using single-walled carbon nanotubes targeted by covalently-coupled monoclonal antibodies," International Journal of Cancer, vol. 125, no. 12, pp. 2970-2977, 2009.

[105] H. Park, J. Yang, J. Lee, S. Haam, I.-H. Choi, and K.-H. Yoo, "Multifunctional nanoparticles for combined doxorubicin and photothermal treatments," ACS Nano, vol. 3, no. 10, pp. 29192926, 2009.

[106] N. H. Levi-Polyachenko, E. J. Merkel, B. T. Jones, D. L. Carroll, and J. H. Stewart IV, "Rapid photothermal intracellular drug delivery using multiwalled carbon nanotubes," Molecular Pharmaceutics, vol. 6, no. 4, pp. 1092-1099, 2009.

[107] C. J. Gannon, P. Cherukuri, B. I. Yakobson et al., "Carbon nanotube-enhanced thermal destruction of cancer cells in a noninvasive radiofrequency field," Cancer, vol. 110, no. 12, pp. 2654-2665, 2007.

[108] M. J. O'Connell, S. H. Bachilo, C. B. Huffman et al., "Band gap fluorescence from individual single-walled carbon nanotubes," Science, vol. 297, no. 5581, pp. 593-596, 2002.

[109] S. V. Torti, F. Byrne, O. Whelan et al., "Thermal ablation therapeutics based on CNx multi-walled nanotubes," International Journal of Nanomedicine, vol. 2, no. 4, pp. 707-714, 2007.

[110] P. Chakravarty, R. Marches, N. S. Zimmerman et al., “Thermal ablation of tumor cells with antibody-functionalized singlewalled carbon nanotubes," Proceedings of the National Academy of Sciences of the United States of America, vol. 105, no. 25, pp. 8697-8702, 2008.

[111] N. Shao, S. Lu, E. Wickstrom, and B. Panchapakesan, "Integrated molecular targeting of IGF1R and HER2 surface receptors and destruction of breast cancer cells using single wall carbon nanotubes," Nanotechnology, vol. 18, no. 31, Article ID 315101, 2007.

[112] B. Kang, D. Yu, Y. Dai, S. Chang, D. Chen, and Y. Ding, "Cancer-cell targeting and photoacoustic therapy using carbon nanotubes as 'bomb' agents," Small, vol. 5, no. 11, pp. 1292-1301, 2009. 
[113] C. Loo, A. Lowery, N. Halas, J. West, and R. Drezek, "Immunotargeted nanoshells for integrated cancer imaging and therapy," Nano Letters, vol. 5, no. 4, pp. 709-711, 2005.

[114] I. H. El-Sayed, X. Huang, and M. A. El-Sayed, "Selective laser photo-thermal therapy of epithelial carcinoma using anti-EGFR antibody conjugated gold nanoparticles," Cancer Letters, vol. 239, no. 1, pp. 129-135, 2006.

[115] Z. Liu, W. B. Cai, L. N. He et al., "In vivo biodistribution and highly efficient tumour targeting of carbon nanotubes in mice," Nature Nanotechnology, vol. 2, no. 1, pp. 47-52, 2007.

[116] J. Meng, J. Duan, H. Kong et al., "Carbon nanotubes conjugated to tumor lysate protein enhance the efficacy of an antitumor immunotherapy," Small, vol. 4, no. 9, pp. 1364-1370, 2008.

[117] C. H. Villa, T. Dao, I. Ahearn et al., "Single-walled carbon nanotubes deliver peptide antigen into dendritic cells and enhance IgG responses to tumor-associated antigens," ACS Nano, vol. 5, no. 7, pp. 5300-5311, 2011. 

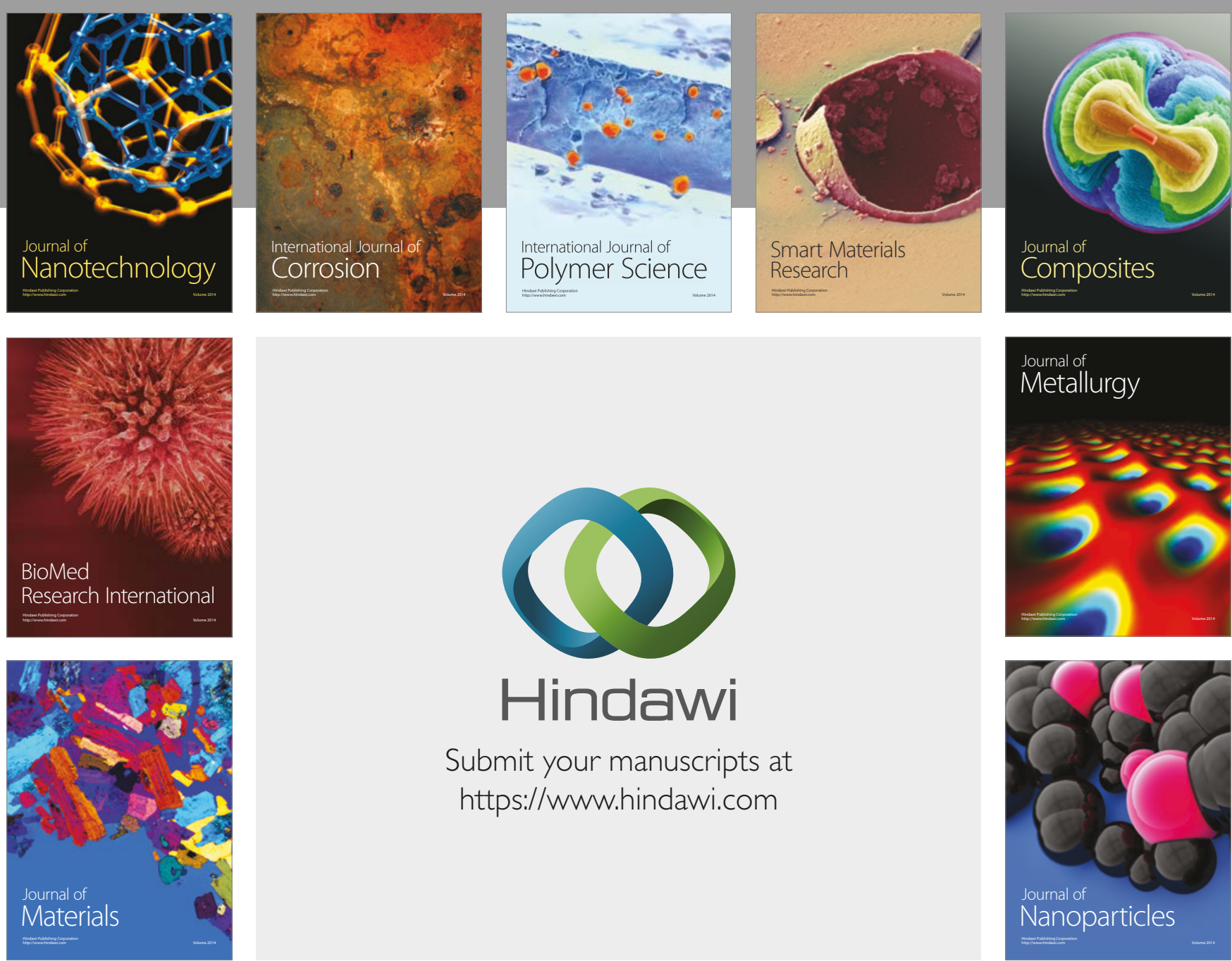

\section{Hindawi}

Submit your manuscripts at

https://www.hindawi.com
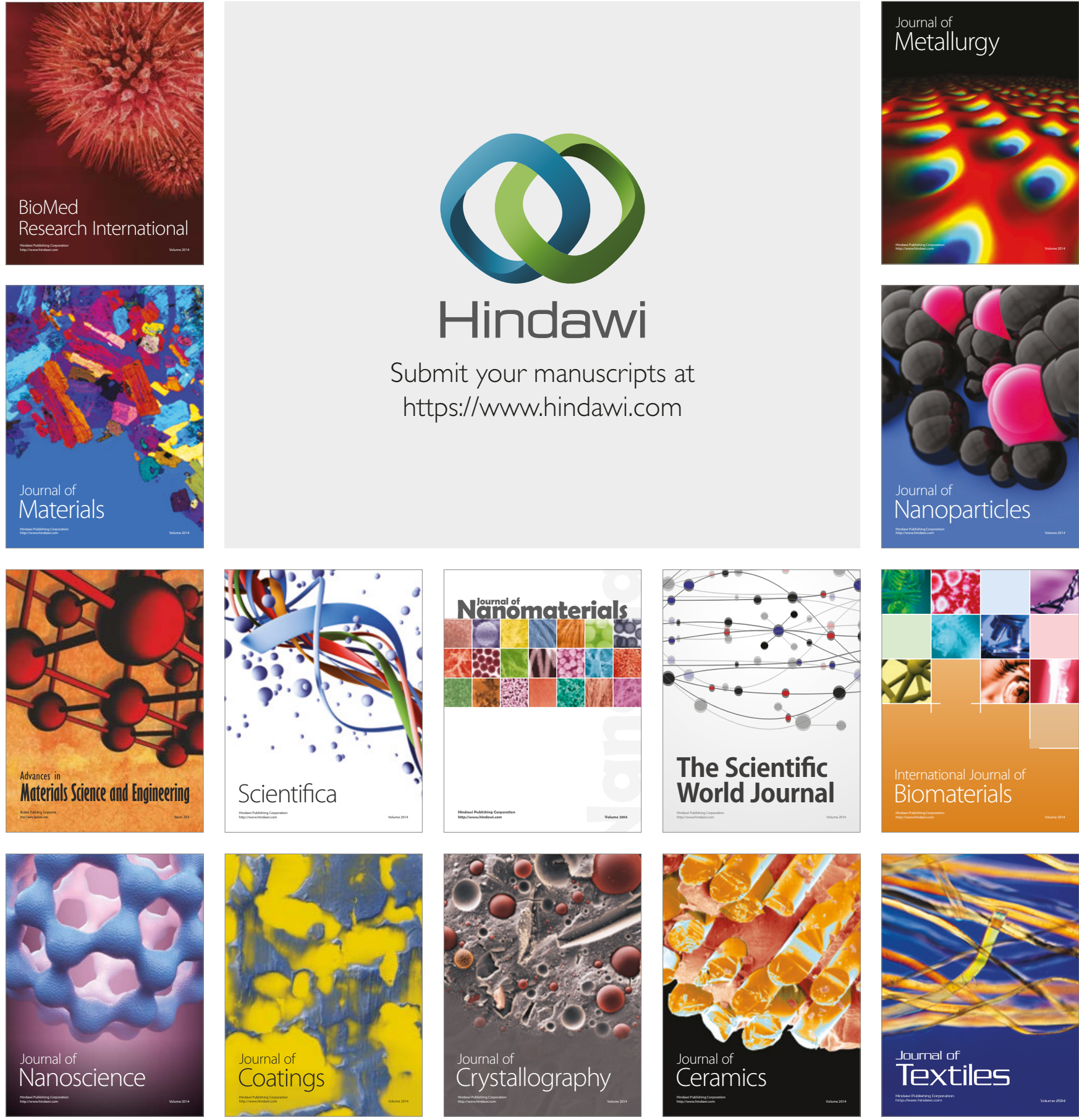

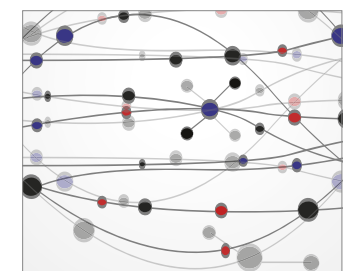

The Scientific World Journal
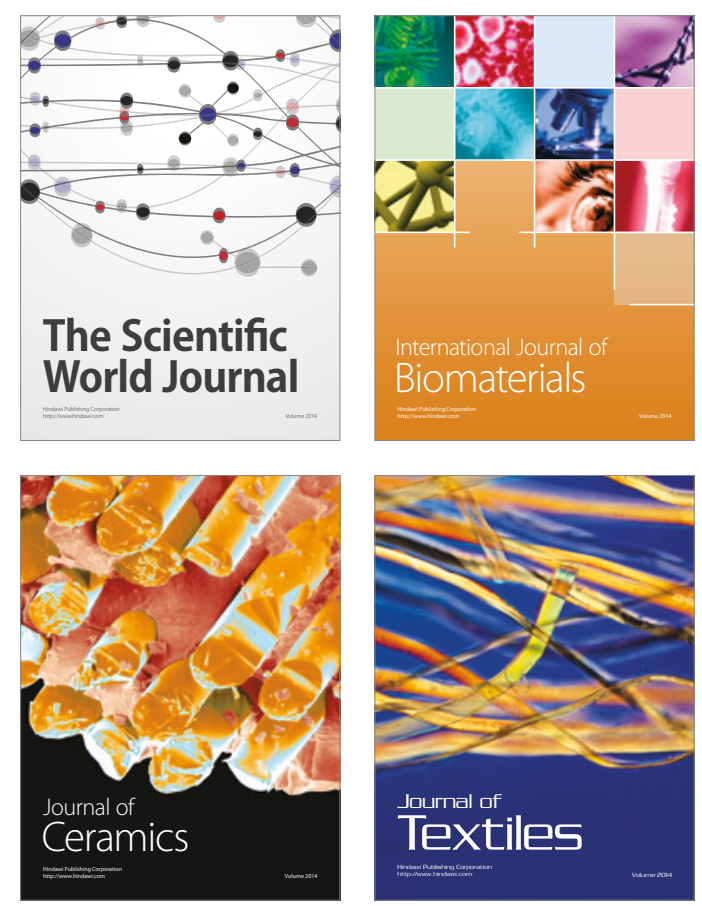